\title{
How can we strengthen pathology services in Cambodia?
}

\author{
Hiroki Akaba1, Noriko Fujita ${ }^{1, *}$, Gerhard Stauch², Yasuyo Matsumoto', Tomoko Wakasa ${ }^{3}$, Kunimitsu Kawahara ${ }^{4}$, \\ Motoji Sawabe ${ }^{5}$ Toshiaki Kawai ${ }^{6}$ \\ ${ }^{1}$ Bureau of International Health Cooperation, National Center for Global Health and Medicine, Tokyo, Japan; \\ ${ }^{2}$ Consultant in Telepathology, Aurich, Germany; \\ 3 Department of Diagnostic Pathology, Kindai University Nara Hospital, Nara, Japan; \\ ${ }^{4}$ Department of Pathology, Osaka Habikino Medical Center, Osaka, Japan; \\ ${ }^{5}$ Department of Molecular Pathology, Graduate School of Medical and Dental Sciences, Tokyo Medical and Dental University, Tokyo, Japan; \\ ${ }^{6}$ Toda Central Medical Laboratory, Saitama, Japan.
}

\begin{abstract}
Rapid economic growth and a changing disease burden have increased the demand for pathology services in Cambodia. This paper describes the status of pathological services and international support for pathology professionals in Cambodia, and discusses future needs for strengthening pathology services. In 2016, there were only four pathologists and 18 pathology technologists in Cambodia. A postgraduate course in pathology was created in 2015, and five residents became certified in 2018. Besides multinational support with lectures and practice for pathologists, the Japanese team provides on-the-job training for pathology technologists to improve slide preparation for diagnosis. A clinicopathological conference was introduced to strengthen the communication among pathologists, pathology technologists, and gynecologists. Although there is a long way to go to reach high quality pathological services, coordination among international partners needs to continue, as does the balance between human resource development for pathology professionals, to provide a higher level of care to local citizens.
\end{abstract}

Keywords: Pathologist, pathology technologist, human resource development, cervical cancer

\section{Introduction}

Today, the prevalence of major non-communicable diseases (NCDs) in Cambodia has been increasing. Among the increased disease burden of NCDs in Cambodia, cervical cancer was the leading cause of female cancers, which was estimated at 767 among the entire population, 16 million in 2014 (1), exceeding the maternal mortality rate in 2015 , which stood at 590 (2). With an urgent need for action, cervical cancer was elevated to a disease to be given priority, focusing on prevention, screening, and treatment services (3).

To respond to the cervical cancer problem, in 2015 the Cambodian Society of Gynecology and Obstetrics started a joint project on cervical cancer with the Japan Society of Gynecology and Obstetrics. Following the World Health Organization (WHO) recommendation of a comprehensive approach (4), a primary cervical cancer screening with testing for the high-risk human papillomavirus, followed by a secondary screening with a colposcopy and early treatment for positive cases, were successfully introduced (5). WHO announced in 2018 a global call to action towards the elimination of cervical cancer, and called for all stakeholders to unite behind this common goal. However, the major obstacle in scaling up a cervical cancer screening program in Cambodia was identified as the extremely limited capacity of its pathological services. When any screening program is scaled up in a country, there will inevitably be a large increase in the detection rate of the target cancer. The national capacity for pathological diagnosis and the treatment of cancer therefore need to be considered in parallel.

This paper aims to describe the status of pathological services and international support for pathology professionals since 2017 in Cambodia, and to discuss future needs for strengthening pathology services.

\section{The situation of pathology services in Cambodia}

In 2016, for the population of 16 million Cambodians, there were only four pathologists and 18 pathology technologists available. After five residents completed a postgraduate course discussed below, the number of pathologists increased to nine in 2018. Thereafter, some certified pathologists went abroad to get further training, therefore, eight pathologists actually work in Cambodia at the present time (Table 1).

Considering pathology laboratories in the public sector, there are three national hospitals with pathology 
Table 1. Pathological services in national hospitals in Phnom Penh, Cambodia

\begin{tabular}{|c|c|c|c|c|}
\hline Items & Calmette Hospital & Kossamak Hospital & KSFH & $\mathrm{NMCHC}$ \\
\hline \multicolumn{5}{|l|}{ Number of medical staff } \\
\hline Pathologists* & 2 & 1 & 3 & 1 \\
\hline Pathology technicians & 5 & 3 & 6 & 2 \\
\hline \multicolumn{5}{|l|}{ Staining methods } \\
\hline Papanicolaou & + & + & + & + \\
\hline Hematoxylin eosin & & + & + & \\
\hline Hematoxylin phloxine saffron & + & & & \\
\hline Giemsa & + & + & + & \\
\hline Grocott & + & & & \\
\hline Periodic acid Schiff & + & + & + & \\
\hline Masson trichrome & + & & & \\
\hline Ziehl Neelsen & + & + & & \\
\hline Iron stain & + & & & \\
\hline Alcian Blue & + & & & \\
\hline
\end{tabular}

*There is another pathologist working in national university. Abbreviation: KSFH, Khmer Soviet Friendship Hospital; NMCHC, National Mother and Child Health Center.

services, and one national hospital with cytology services. Three national hospitals deal with approximately 4,000 histology specimens annually, and only one of them is capable of performing immunohistochemistry. However, as there are several private laboratories in which specialists provide pathology services as a dual practice, it is difficult to precisely estimate the actual number of pathology services provided in Cambodia.

The University of Health Sciences (UHS), established in 1946, has been the first and only national medical school that has a department of anatomical pathology. Responding to the increased demand for pathological services, a postgraduate course in Pathology was created at UHS in 2015, and six doctors entered. However, it was difficult to accomplish the curriculum because of insufficient numbers of Cambodian lecturers and institutes for clinical practice. Although overseas training is part of the curriculum, UHS faced the difficulty of identifying appropriate institutions to receive Cambodian postgraduate students. The Technical School for Medical Care under UHS served as the leading institute for laboratory technologists. Since there are no pathology classes in the pre-service educational curriculum, current pathological technologists learned the techniques on-site in the hospital pathology department.

\section{Intervention by international supporters}

\section{Pathologists}

Lectures and overseas training for the post-graduate course were covered by volunteer pathologists from France, Germany, Japan, Singapore, and Australia. In addition, overseas training was supported by the German and Japanese team. As a result, the first batch of five residents became certified and the number of pathologists in Cambodia rose to nine in 2018. The second batch of postgraduate courses in Pathology will be opened in
2020 contributing the specialists' education to serve to ensure the quantity and quality of the pathologists.

The German and European groups of pathologists introduced the iPath network (6) of telemedicine pathology consultation, and a weekly Skype teaching session. The Japanese team has supported regular clinicopathological conferences between pathologists and gynecologists since 2018 to strengthen communication between pathologists and clinicians. In 2017 the Japanese team also invited the existing four Cambodian pathologists to share Japan's education system and the functions of the professional society. From individual and fragmented support to more complementary and integrated ones, meetings to communicate with UHS and its supporters started in 2017 among a small community of pathologists in Cambodia.

\section{Pathology technologists}

Although basic pathology equipment and supplies were available, slides were often difficult to read due to inadequate preparation. Therefore, step-bystep training has been offered to technologists for improving the quality of slide preparation by a team of Japanese pathology technologists since 2017. In the beginning, four Cambodian technologists were invited for technical training in Japan, and their skills improved enough to prepare good-quality slides in Japan, where laboratories have no resource constraints on reagents and consumables. As a next step, the technical support mainly focused on two activities: developing Standard Operating Procedures (SOP) for adapting the Cambodians' process of embedding, sectioning, and staining; and developing a self-evaluation form to evaluate slides for quality improvement.

Future perspective responding to the demands of pathology services 
Table 2. The number of pathology professionals in Asian, African, and high-income countries

\begin{tabular}{|c|c|c|c|c|c|}
\hline Country & Population ( 8 ) & $\begin{array}{c}\text { GDP per } \\
\text { Capita ( } 8)\end{array}$ & $\begin{array}{l}\text { Population } \\
\text { per pathologist }\end{array}$ & Pathologists & $\begin{array}{c}\text { Pathology } \\
\text { Technologists }\end{array}$ \\
\hline \multicolumn{6}{|l|}{ Asia } \\
\hline Cambodia & $16,486,542$ & 4,018 & $2,060,818$ & 8 & 18 \\
\hline Lao PDR* & $7,169,455$ & 7,038 & 796,606 & 9 & 7 \\
\hline Vietnam (9) & $96,462,106$ & 6,790 & 263,558 & 366 & NA \\
\hline Malaysia (10) & $31,949,777$ & 29,551 & 85,886 & 372 & NA \\
\hline \multicolumn{6}{|l|}{ Africa (11) } \\
\hline Chad & $15,946,876$ & 1,945 & $7,973,438$ & 2 & 2 \\
\hline Malawi & $18,628,747$ & 1,205 & $2,069,861$ & 9 & 2 \\
\hline Senegal & $16,296,364$ & 3,458 & $2,328,052$ & 7 & NA \\
\hline Zambia & $17,801,030$ & 4,033 & $2,966,838$ & 6 & 6 \\
\hline Zimbabwe & $14,645,648$ & 2,434 & $2,929,130$ & 5 & 1 \\
\hline \multicolumn{6}{|l|}{ High-income countries } \\
\hline Japan (12) & $126,860,301$ & 42,067 & 49,965 & 2,539 & NA \\
\hline United States (13) & $329,064,917$ & 59,928 & 25,630 & 12,839 & NA \\
\hline United Kingdom (14) & $67,530,172$ & 44,920 & 46,766 & 1,444 & NA \\
\hline
\end{tabular}

Data source: References (8-14). *personal communication

Human resources capacity building of pathology professionals with focus not only for pathologists but also pathology technicians

According to the calculation of required human resources for pathological services presented by Sayed et al. (7), the minimum pathology and laboratory medicine staffing needs are one general pathologist, six pathology technologists, and one pathology assistant for every 50,000-200,000 people, for typical surgical procedure levels: basic trauma surgery, general surgery, emergency obstetrics and gynecology, and surgery by some specialists. With a population of approximately 16 million in Cambodia, 71 more pathologists and 460 more technologists are needed to meet the recommended level.

Table 2 shows the number of pathology professionals in Asian, African, and high-income countries (8-14). There is a huge gap between low- and middle- income countries and high-income countries. In Southeast Asian countries, Cambodia and Lao People's Democratic Republic have scarce human resources, similar to African countries. Notably all pathological laboratories are only in the capital city.

Although there is a long way to go to develop human resources to achieve qualified pathological services for surgical diagnosis in Cambodia, the pathology technologist workforce should be prioritized. With improved transportation, if pathology technologists are able to make slides in rural areas, it is possible for pathologists to make a diagnosis in the national hospitals. Furthermore, as it is predicted that telepathology technology could be installed in low- and middleincome countries, if all the specimens were of high quality suitable for telepathology, pathologists could make a diagnosis wherever they are (15). Since the postgraduate pathology course has only recently started, qualified pathology technologists are urgently needed for pathology services in Cambodia.

\section{Coordination with international donors and national stakeholders}

In a country with scarce human resources like Cambodia, a short-term visitor program is suggested as a common beneficial model, although it has the negative aspects of non-sustainability, intermittency, and dependency on local communities (11). Coordination and collaboration among technical supporters would be imperative for identifying how to effectively fill the gap and build a consensus for moving the country toward sustainability. Setting up a communication mechanism among international supporters with national stakeholders would be applicable for other resourcelimited countries as well.

\section{Golden triangle for sustainable quality management}

Good communication and collaboration among clinicians, technicians, and pathologists would be imperative and could be called the golden triangle to improve the quality of pathology services in Cambodia. Cervical cancer could be an entry point for capacity building of pathology professionals. When the multidisciplinary and multinational supporters communicate thoroughly, we can identify appropriate resources and opportunities to effectively fulfill the gap of pathology services in Cambodia.

No matter how many pathology technicians are trained, it is still important to evaluate the quality of the specimens. Although SOP and the "evaluation sheet" have been introduced in each department to maintain the quality of slides, these schemes must be revised continuously as the quality management cycle in Cambodia. 


\section{Strengthening infrastructure}

Inadequate infrastructure such as the supply chain of reagents and maintenance of the equipment is often the major problem in laboratories in low-and-middle income countries, but often neglected. While the number of pathological examinations is increasing, an unstable supply of reagents, which does not meet the demand will result in a deteriorated quality of specimens, and clinicians. Human resources for the maintenance of medical equipment and devices can also be essential to keep laboratories functioning because they often use second-hand equipment for pathological examinations, which sometimes need to be repaired. Therefore, in order to achieve an accurate pathological diagnosis, strengthening infrastructure is an essential issue, and needs to be solved among Cambodians in the long run.

In conclusion, rapid economic growth and a changing disease burden has increased the demand for pathology services, with more clinicians expected for improving quality of pathology services in Cambodia. Although there is a long way to go to achieve quality pathological services for surgical diagnosis in Cambodia, coordination among international partners is essential, as is empowerment of the golden triangle among pathologists, technicians and clinicians. In addition, continuous quality management plays an important role to deliver sustainable high-quality pathological services to provide a higher level of care to local citizens.

\section{Acknowledgements}

We would like to thank Prof. Gilbert Burnham at Johns Hopkins Bloomberg School of Public Health for his constructive criticism for finalizing the manuscript. We would also like to express our sincere gratitude to the many Cambodian and Japanese doctors and technologists who have supported this project.

Japanese interventions are funded by the Ministry of Health, Labor and Welfare (Projects for global growth of medical technologies, systems and services through human resource development from 2017 until 2019).

\section{References}

1. World Health Organization. Cancer country profile, Cambodia 2014. https://www.who.int/cancer/countryprofiles/khm_en.pdf (accessed October 20, 2019).

2. World Health Organization. Maternal mortality in 19902015, Cambodia. https://www.who.int/gho/maternal_ health/countries/khm.pdf (accessed October 20, 2019).

3. Ministry of Health, Cambodia. National Strategic Plan for the Prevention and Control of Noncommunicable Diseases
2013-2020. https://www.iccp-portal.org/system/files/ plans/KHM_B3_NSP-NCD\%202013-2020_Final\%20 approved.pd $\bar{f}$ (accessed October 21, 2019).

4. World Health Organization. Comprehensive cervical cancer control: A guide to essential practice. WHO Library Cataloguing-in-Publication Data. 2014. https://apps.who. int/iris/bitstream/handle/10665/144785/9789241548953_ eng.pdf? sequence $=1$ (accessed October 21, 2019).

5. Ueda Y, Kawana K, Yanaihara N, et al. Development and evaluation of a cervical cancer screening system in Cambodia: A collaborative project of the Cambodian Society of Gynecology and Obstetrics and Japan Society of Obstetrics and Gynecology. J Obstet Gynaecol Res. 2019; 45:1260-1267.

6. iPath-Network. https://www.ipath-network.com/ipath/ (accessed October 23, 2019).

7. Sayed S, Cherniak W, Lawler M, Tan SY, El Sadr W, Wolf N, Silkensen S, Brand N, Looi LM, Pai SA, Wilson ML, Milner D, Flanigan J, Fleming KA. Improving pathology and laboratory medicine in low-income and middle-income countries: roadmap to solutions. Lancet. 2018; 391:1939-1952.

8. World meters. https://www.worldometers.info (accessed November 1, 2019)

9. Duong VD. Pathology and telepathology in Vietnam. Computational Pathology and Telepathology: SY05-2. Pathology. 2014; 46:S8.

10. National Healthcare statistics initiative, $\mathrm{MOH}$ Malaysia. http://www.crc.gov.my/nhsi/charts/malaysia_doctor.php (accessed November 3, 2019)

11. Nelson AM, Milner DA, Rebbeck TR, Iliyasu Y. Oncologic care and pathology resources in Africa: survey and recommendations. J Clin Oncol. 2016; 34:20-26.

12. The Japan Society of Pathology. Certified pathologists. http://pathology.or.jp/senmoni/board-certified.html (accessed November 5, 2019)

13. Metter DM, Colgan TJ, Leung ST, Timmons CF, Park JY. Trends in the US and Canadian pathologist workforces from 2007 to 2017. JAMA Netw Open. 2019; 2:e194337.

14. The Royal College of Pathologists, the pathology workforce https://www.rcpath.org/uploads/ assets/952a934d-2ec3-48c9-a8e6e00fcdca700f/MeetingPathology-Demand-Histopathology-WorkforceCensus-2018.pdf (accessed November 5, 2019)

15. Voelker HU, Stauch G, Strehl A, Azima Y, MuellerHermelink HK. Diagnostic validity of static telepathology supporting hospitals without local pathologists in low-income countries. J Telemed Telecare. 2018; doi.10.1177/1357633X18818745.

Received October 30, 2019; Revised November 18, 2019; Accepted November 28, 2019

*Address correspondence to:

Noriko Fujita, Bureau of International Health Cooperation, National Center for Global Health and Medicine, 1-21-1 Toyama Shinjuku-ku, Tokyo 162-8655, Japan.

E-mail: norikof@it.ncgm.go.jp 